\title{
ESTIMATIVA DO TORQUE MUSCULAR DE EXTENSORES DO JOELHO DE IDOSOS BASEADO EM TESTES DE DESEMPENHO FÍSICO FUNCIONAL
}

\author{
ESTIMATE OF MUSCLE TORQUE OF ELDERLY KNEE EXTENSORS \\ BASED ON PHYSICAL FUNCTIONAL PERFORMANCE TESTS
}

\author{
Keuly Garcia da Silva ${ }^{1,2}$ \\ keulygarcia@gmail.com \\ Lucas Bet da Rosa Orssatto \\ lucasorssatto@gmail.com \\ Iris Natália Mendonça Barros ${ }^{4}$ \\ iris_natalia16@hotmail.com \\ Kayth Sousa Nascimento ${ }^{2}$ \\ kayth.nascimento@gmail.com \\ Inês Amanda Streit ${ }^{2,5}$ \\ inesamanda@gmail.com \\ iD Ewertton de Souza Bezerra ${ }^{1,2,5}$ \\ ewertton_bezerra@ufam.edu.br
}

Número do Parecer do Comitê de Ética 3.139.675

Autor correspondente

Ewertton de Souza Bezerra

Ewertton_bezerra@ufam.edu.br

Endereço para correspondência:

Faculdade de Educação Física e Fisioterapia- Universidade Federal do Amazonas. Av. General Rodrigo Octavio Jordão Ramos, 1200 -

Coroado I, Manaus - AM, 69067-005.

${ }^{1}$ Laboratório de Estudos do Desempenho Humano, Faculdade de Educação Física e Fisioterapia, Universidade Federal do Amazonas, Manaus-AM, Brasil.

${ }^{2}$ Projeto de Atividade Física para Idosos, Faculdade de Educação Física e Fisioterapia, Universidade Federal do Amazonas, ManausAM, Brasil.

${ }^{3}$ School of Exercise and Nutrition Sciences, Queensland University of Technology, Brisbane, Queensland, Australia.

${ }^{4}$ Universidade do Estado do Amazonas, Manaus-AM, Brasil.

${ }^{5}$ Programa de Pós Graduação Stricto Sensu em Ciências do

Movimento Humano, Faculdade de Educação Física e Fisioterapia,

Universidade Federal do Amazonas, Manaus-AM, Brasil.

\begin{abstract}
Resumo
Objetivo: Verificar quais métodos de avaliação clínicos de força e potência muscular, e testes de desempenho físico funcionais estão correlacionados com o pico de torque isométrico e dinâmico dos extensores de joelho, e desenvolver equações preditivas que estimem o pico de torque isométrico e dinâmico em pessoas idosas.

Métodos: Foram selecionados 49 sujeitos ( $\geq 60$ anos) de ambos os sexos. O pico de torque muscular isométrico ( $\mathrm{PT}_{\mathrm{ISO}}$ ) e dinâmico concêntrico ( $\mathrm{PT}_{\mathrm{CON}}$ ) dos extensores da articulação do joelho do membro preferido foi avaliado através de dinamômetro isocinético no primeiro dia de avaliação. Quarenta e oito horas depois o teste força máxima (1-RM) unilateral do membro preferido foi executado na cadeira extensora, seguido do teste de potência do membro superior com uma medicine ball de $2 \mathrm{~kg}$ (ABM-2). No terceiro dia foram avaliados o teste de equilíbrio dinâmico (time up and go), a capacidade de subir e descer escadas (separadamente), com estes três testes foi construído um índice funcional (IFUNC).

Resultados: Os principais resultados demonstraram que o IFUNC não prediz melhoria no pico de torque extensor do joelho $(\mathrm{p}>0,05)$. Contrapondo a hipótese inicial que alterações no desempenho do pico de torque, tanto isométrico, como dinâmico, teria uma resposta direta com o desempenho funcional.

Conclusão: As medidas de força máxima para membro inferior (cadeira extensora-1$\mathrm{RM}$ ) e potência de membro superior (arremesso da bola de medicine ball 2-kg) quando associadas explicam com mais de $60 \%(\mathrm{p}<0,05)$ uma mudança no desempenho na força isométrica e dinâmica dos extensores de joelho de idosos não treinados.
\end{abstract}

Palavras-chave: Envelhecimento. Músculo esquelético. Dinamômetro de força muscular.

\begin{abstract}
Objective: The aim of the present study was to verify which clinical strength assessment methods and physical functional performance tests are correlated with the isometric and dynamic peak torque of the knee extensors, and to develop predictive equations that estimate the isometric and dynamic peak torque in elderly people. Methods: Forty-nine subjects ( $\geq 60$ years old) of both sexes were selected. The peak isometric torque ( $\mathrm{PT}_{\mathrm{ISO}}$ ) and concentric dynamic torque ( $\left.\mathrm{PT}_{\mathrm{CON}}\right)$ of the knee joint extensors on the preferred limb was assessed using an isokinetic dynamometer on the first day of assessment. Forty-eight hours later the unilateral maximum strength test (1$\mathrm{RM}$ ) of the preferred limb was performed on the leg extension exercise, followed by the upper limb power test with a $2 \mathrm{~kg}$ medicine ball (ABM-2). On the third day, the dynamic balance test (time up and go), the ability to climb and descend stairs (separately) were evaluated, with these three tests a functional index (IFUNC) was built. Results: The main results demonstrated that the IFUNC does not predict improvement in the peak knee extensor torque ( $p>0.05$ ). Against the initial hypothesis which improves the performance of peak torque, so much isometric and dynamic, would have a direct response to functional performance.

Conclusion: The measures of maximum strength for the lower limb (leg extension, 1$\mathrm{RM}$ ) and power of the upper limb (throwing the medicine ball 2-kg) when associated explain more than $60 \%(\mathrm{p}<0.05)$ changes on performance in the isometric and dynamic strength of knee extensors of untrained elderly.
\end{abstract}

Keywords: Aging. Muscle, skeletal. Muscle strength dynamometer.

\section{Cite como}

Vancouver

Silva, KG, Orssatto, LBR, Barros, INM, Nascimento, KS, Streit, IA, Bezerra, ES. Estimativa do torque muscular de extensores do joelho de idosos baseado em testes de desempenho físico funcional. Conscientiae Saúde 2020;19(1):1-17, e18247. https://doi.org/10.5585/conssaude.v19n1.18247. 


\section{Introdução}

A força produzida durante as contrações máximas e submáximas, realizadas pelos músculos esqueléticos, é controlada pela variação do número de unidades motoras recrutadas e pela taxa de descarga dos potenciais de ação, que inervam cada unidade motora ativa (1). Alterações do sistema neuromuscular relacionadas à idade têm um efeito marcante nessa capacidade de produção de força muscular. São observados comprometimentos em diferentes etapas dessa produção, como no sistema nervoso central, que coordena essas ações; no sistema nervoso periférico, que transporta as informações para o músculo esquelético; e no sistema muscular, que realizará a contração para geração de força (2) .

Estudos transversais, que investigaram os músculos dos membros inferiores, indicam que a força é geralmente reduzida em $10 \%$ por década, a partir de aproximadamente 40-50 anos de idade, com evidências de declínios acelerados em idade muito avançada (2). Além disso, rápidas velocidades de encurtamento apresentem maior redução do que velocidades mais baixas; embora tais diferenças possam variar entre os grupos musculares (3). Adicionalmente, tal redução é refletida negativamente no torque máximo durante as contrações dinâmicas isocinéticas em idade avançada, sem distinção entre os sexos (4).

Tais reduções da força muscular, decorrente do envelhecimento, acarretam na redução da capacidade do desempenho físico funcional em realizar atividades básicas da vida diária (e.g., subida de escada, deambulação e tempo de levantar de uma cadeira) (2). Por exemplo, o componente potência está fortemente relacionado às tarefas de desempenho físico funcional, sendo assim, torna-se excelente preditor da função físico funcional, e por consequência, um indicador da incapacidade física em idosos (5). Dessa maneira, faz-se essencial monitorar a força muscular em idosos acompanhar o nível de comprometimento e conseguir prescrever intervenções mais adequadas. Isso porque, a atividade física pode modificar as propriedades e funções da unidade motora em homens e mulheres idosos (6). Tarefas mais complexas e exigentes, como aquelas que requerem maior intensidade ou coordenação, podem esclarecer melhor as diferenças entre idosos saudáveis com baixa e alta capacidade funcional (7).

Desenvolver modelos que possam propor a avaliação da força muscular isométrica e dinâmica de membros inferiores de idosos, partindo de TESTE CLÍNICOS que apresentam demanda de desempenho físico funcional, de baixo custo, fácil aplicação e com alta reprodutibilidade torna-se de grande aplicação para profissionais de saúde (por exemplo, personal trainers, fisioterapeutas, nutricionistas e reumatologistas). Sendo assim, os objetivos do presente estudo foram: (1) verificar quais métodos de avaliação de força muscular (i.e. carga 
máxima com equipamento convencional e potência para membros superiores) e teste funcionais (i.e subir e descer escadas, timed up and go) estão correlacionados com o pico de torque isométrico e dinâmico dos extensores de joelho e (2) desenvolver equações preditivas que estimem o pico de torque isométrico e dinâmico de pessoas idosas com base nos referidos testes. Nossa hipótese inicial é que testes de desempenho físico funcionais associados são capazes de predizer respostas em testes realizados no isocinético (pico de torque isométrico e concêntrico), sem haver necessidade de associar com testes de força e potência muscular mais clínicos, como uma repetição máxima (1-RM) do membro inferior e arremesso da bola de medicine ball para membro superior.

\section{Métodos}

\section{Procedimentos experimentais}

O presente estudo caracterizou-se quanto à natureza como uma pesquisa quantitativa, aplicada e transversal. Foi realizado em cinco dias não consecutivos, separados por pelo menos 48 horas. Inicialmente, após a seleção dos participantes, foi realizada uma sessão de familiarização com o exercício cadeira extensora que seria realizado no teste de 1-repetição máxima (1-RM). Em seguida, em dois dias, foi aplicado o teste e reteste de 1-RM. Posteriormente, no quarto dia, o teste no isocinético. E, por fim, no último dia, os testes de desempenho físico funcional e potência de membro superior.

\section{Participantes do estudo}

Após o preenchimento de questionários específicos de histórico de saúde e de atividade física, foram selecionados 49 sujeitos (39 mulheres e 10 homens, 65,6 4,7 anos). Essa seleção foi realizada de acordo com os seguintes critérios de inclusão: idade acima de 60 anos, ser fisicamente independente, sem prática regular de exercício resistido nos últimos 12 meses, livre de doença cardíaca e livre de disfunção ortopédica. O tamanho da amostra foi baseada no proposto por Miles; Shevin (8) considerando um poder de $80 \%$, em que o tamanho do efeito está relacionado com o número de previsores. O consentimento informado por escrito foi obtido de todos os participantes após uma descrição detalhada dos procedimentos do estudo. Todos os procedimentos realizados neste estudo foram aprovados por um Comitê de Ética Institucional local com o parecer $n^{\circ}$ 01579418.6.0000.5020 e seguiram as diretrizes éticas da Declaração de Helsinque ( $64^{\circ}$ WMA General Assembly, Fortaleza, Brasil, outubro, 2013). 


\section{Medidas de desempenho motor}

\section{Pico de torque isométrico e dinâmico}

Para a avaliação do torque muscular dos extensores da articulação do joelho foi utilizado o dinamômetro Isocinético Biodex System 4 Pro® (Biodex Medical Systems Inc., Shirley, NY, USA). O pico de torque foi avaliado durante as contrações voluntárias isométrica e dinâmica máximas. Antes de iniciar os testes, os participantes foram posicionados no dinamômetro e o membro avaliado, assim como o tronco e a região pélvica foram estabilizados utilizando tiras não elásticas, a fim de evitar movimentos compensatórios. $\mathrm{O}$ banco foi posicionado em $85^{\circ}$ de flexão de quadril e $70^{\circ}$ de flexão de joelho $\left(0^{\circ}=\right.$ posição neutra do quadril e extensão total do joelho). O membro preferido foi avaliado seguindo o critério aplicado no questionário de Waterloo (9).

Após o posicionamento, foi realizado um aquecimento isocinético, com 10 repetições de contrações concêntricas de extensão-flexão do joelho, em amplitude de movimento previamente determinada $\left(20^{\circ}\right.$ a $\left.100^{\circ}\right)$ a uma velocidade angular de $120 \%$ s. Seguiu-se a execução de 3 séries de contrações isométricas submáximas de extensão do joelho, na posição de $70^{\circ}$. Para as contrações isométricas máximas, os participantes foram instruídos a produzir força “o mais rápido e mais forte possível”, com ênfase em um início rápido para a contração. O encorajamento verbal foi dado antes e durante todo o teste, usando o termo "mais força" (10). Para definir o valor de pico de torque isométrico (PTISO), uma média entre três tentativas foi feita a partir dos valores obtidos durante o exame. Em seguida, adotando os mesmos procedimentos, o pico de torque durante a ação concêntrica $\left(\mathrm{PT}_{\mathrm{CON}}\right)$ foi determinado para a velocidade de $60 \%$ s (11). Três contrações consecutivas foram aplicadas e a média delas foi o valor adotado para as análises finais (12).

\section{1 repetição máxima $(1-R M)$}

Anteriormente às sessões de teste e reteste de uma repetição máxima (1-RM), foi realizada uma sessão de familiarização com o exercício cadeira extensora. Após 24 h da sessão de familiarização, realizou-se a primeira sessão do teste de 1-RM e 48h após foi aplicado o reteste, apresentando um coeficiente de correlação intraclasse de 0,93 . Previamente à realização do teste de 1-RM, cada participante foi submetido a 5 minutos de aquecimento geral (esteira ergométrica) e específico com regressão de 10, 6 e 3 repetições com cargas submáximas. O protocolo do teste de 1-RM seguiu as recomendações de Brown e Weir (13). Para minimizar erros durante a aplicação do teste, as seguintes estratégias foram adotadas: a) padronizar a 
explicação dada aos participantes antes da realização do teste; b) encorajamento verbal durante a realização das contrações; c) adoção de no máximo cinco tentativas com cinco minutos de intervalo entre elas; d) para não haver influência do conhecimento da carga durante o teste, a torre de placas da cadeira extensora foi ocultada. A maior carga alcançada entre os dois dias de teste foi considerada a carga de 1-RM da cadeira extensora e utilizada nas análises finais. Durante os dias de teste, os participantes foram instruídos a não realizar nenhum exercício.

\section{Potência de membros superiores}

O desempenho de arremesso da bola (AMB) foi testado com uma medicine ball de $2 \mathrm{~kg}$ (diâmetro = 0,60 m). Cada participante sentou-se no chão (sobre um colchonete), com a região do tronco posterior apoiada em uma parede e segurou a bola à frente do peitoral com ambas as mãos. Eles foram instruídos a jogar a bola o mais longe e mais rápido possível. A rotação e flexão do tronco e quadril não foram permitidas. Três tentativas foram feitas com intervalos de descanso de um minuto cada, para assegurar que a fadiga ou os efeitos de aprendizagem não influenciassem o desempenho. A distância máxima de lançamento foi determinada usando uma fita de aço flexível, previamente fixa ao chão e perpendicular à parede, que foi usada como encosto para o teste. A média das três tentativas foi usada para análise posterior (14).

\section{Desempenho físico funcional}

O teste timed up and go (TUG) $(2,44 \mathrm{~m})$ foi aplicado para medir o equilíbrio dinâmico (15). Para o TUG, os participantes foram solicitados a iniciar sentados, com os pés totalmente apoiados sobre uma superfície rígida, numa cadeira de encosto de $43 \mathrm{~cm}$ de altura, com os braços cruzados na altura dos ombros. Ao sinal do avaliador, eles foram instruídos a levantarem-se, permanecendo com os braços cruzados, até total extensão do joelho. Em seguida, deslocaram-se por um trajeto de $2,44 \mathrm{~m}$ até um cone, dando a volta e retornando ao ponto de saída, sentando novamente com os braços cruzados a frente do peitoral (16). Foram aplicadas três tentativas com um intervalo mínimo de 30s, a média delas foi usada para análise.

$\mathrm{O}$ teste de subir e descer escadas analisa a capacidade combinada de força e potência e equilíbrio dos membros inferiores (17). Sendo assim, os participantes subiram 8 degraus (de 16 $\mathrm{cm}$ de altura) de maneira segura e usando o corrimão, numa velocidade habitual. O tempo iniciou quando o primeiro pé saiu do chão ao comando do avaliador e foi finalizado quando ambos os pés tocarem o oitavo degrau. Após intervalo de 30s, foi solicitado que o avaliado descesse as escadas. Critérios similares ao anterior, foram usados para contagem do tempo da 
tarefa. Foram aplicadas três tentativas e a média delas foi usada para a análise, as duas ações (subir e descer) foram avaliadas de forma independentes.

Os testes de TUG, subir e descer escadas foram gravados usando um smartphone com frequência de amostragem de $120 \mathrm{~Hz}$ (Iphone 7, Apple® Inc. Califórnia, EUA) e avaliado, posteriormente, por um examinador experiente com software especializado (Kinovea®, França) (18). A média das três tentativas foi utilizada para análises finais. Devido a inter-relação dos três testes (TUG, subir e descer escadas) foi criado um índice funcional (IFUNC) para a análise do modelo de regressão, este consistiu da média dos resultados obtidos nos três testes anteriormente relatados.

\section{Análise estatística}

Os dados são apresentados em média e desvio padrão. A normalidade foi analisada através do teste de Shapiro-Wilk ( $\mathrm{p}>0,05)$. Foram observadas a Correlação de Pearson entre o pico de torque da contração isométrica (PT $\mathrm{PSO}_{\mathrm{SO}}$ e concêntrica ( $\left.\mathrm{PT}_{\mathrm{CON}}\right)$ (variáveis dependentes) e as variáveis independentes (por exemplo, 1-RM, arremesso da bola de medicine ball, IFUNC). A significância foi estabelecida em $p<0,05$. A magnitude da correlação foi analisada como proposto por Cohen (19).

As equações de predição foram desenvolvidas por meio de regressão linear múltipla (ENTER), com as variáveis dependentes (ou seja, $\mathrm{PT}_{\mathrm{ISO}}$ e $\mathrm{PT}_{\mathrm{CON}}$ ) e independentes (1-RM, arremesso da bola de medicine ball, IFUNC). Duas equações de predição foram desenvolvidas para cada variável dependente. Uma análise de validação cruzada foi aplicada através do $\mathrm{R}^{2}$ ajustado, para que pudesse representar a perda de poder de previsão, nesse sentido, utilizou-se a equação de Wherry. Além do mais, um teste de Durbon-Watson analisou a hipótese de independência dos erros e os valores do fator de inflação da variação (FIV) foram calculados para verificar presença de multicolinearidade. Este, quando próximos de 10, indicam presença e quando próximo de 1, indicam ausência de multicolinearidade.

Uma ANOVA analisou se o modelo de regressão é significativamente melhor para prever a saída de $\mathrm{PT}_{\text {ISO }}$ e $\mathrm{PT}_{\mathrm{CON}}$ do que utilizar a média das variáveis previsora. Todas as análises foram feitas no pacote estatístico SPSS para Windows 21.0 (Statistical Package for the Social Science, IBM, Chicago, Ill, USA). 


\section{Resultados}

As variáveis dependentes e independentes estão descritas por sexo dos participantes na Tabela 1.

Tabela 1 - Variáveis adotada no modelo de regressão para ambos os sexos. Média, desvio padrão e coeficiente de variação

\begin{tabular}{ccccc} 
& \multicolumn{2}{c}{ Mulher } & \multicolumn{2}{c}{ Homem } \\
\cline { 2 - 5 } & Média $\pm \mathrm{DP}$ & $\mathrm{CV}(\%)$ & Média $\pm \mathrm{DP}$ & $\mathrm{CV}(\%)$ \\
$\mathrm{PT}_{\text {ISO }}(\mathrm{N} \cdot \mathrm{m})$ & $111,85 \pm 38,42$ & 34,35 & $141,50 \pm 48,64$ & 34,37 \\
$\mathrm{PT}_{\text {CON }}(\mathrm{N} \cdot \mathrm{m})$ & $83,23 \pm 23,97$ & 28,79 & $109,70 \pm 29,78$ & 27,15 \\
$1-\mathrm{RM}(\mathrm{Kg})$ & $16,83 \pm 5,25$ & 31,20 & $25,20 \pm 8,38$ & 33,24 \\
AMB $(\mathrm{m})$ & $2,49 \pm 0,43$ & 17,43 & $3,20 \pm 0,56$ & 17,58 \\
IFUNC (s) & $7,91 \pm 1,63$ & 20,65 & $7,62 \pm 1,55$ & 20,36
\end{tabular}

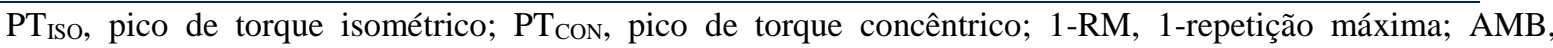
arremesso de medicine ball de 2kg; IFUNC, índice funcional; CV, coeficiente de variação.

Fonte: Própria autora.

As equações preditivas de regressão múltipla, com a variável dependente $\mathrm{PT}_{\text {ISO }}$ são

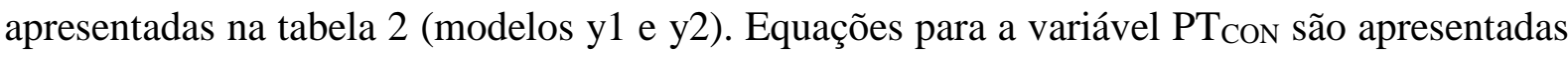
na tabela 3 (modelos y3 e y4). Para todas os modelos, os preditores mostraram correlações superiores a $\mathrm{r}=0,75$.

Tabela 2 - Modelos de equação preditiva para o pico de torque isométrico ( $\mathrm{PT}_{\text {ISO, }}$ modelo y1 e y2) e pico de torque concêntrico a $60 \%$ s ( $\mathrm{PT}_{\mathrm{CON}}$, modelo y3 e y4)

\begin{tabular}{llll}
\hline Equações preditivas & $\mathbf{R}^{2}$ & $\begin{array}{l}\mathbf{R}^{2} \\
\text { ajustado }\end{array}$ & $\begin{array}{l}\text { Durbin- } \\
\text { Watson }\end{array}$ \\
\hline $\begin{array}{l}\mathrm{y} 1=13,93+(-2,30 * \text { IFUNC)+(3,26*1- } \\
\mathrm{RM})+(21,84 * \mathrm{AMB})\end{array}$ & 0,60 & 0,58 & 1,9 \\
$\mathrm{y} 2=-6,94+\left(21,83^{*} \mathrm{AMB}\right)+(3,44 * 1-\mathrm{RM})$ & 0,60 & 0,58 & 1,9 \\
$\mathrm{y} 3=12,26+\left(-0,78^{*} \mathrm{IFUNC}\right)+\left(13,98^{*}\right.$ & 0,68 & 0,65 & 2,2 \\
$\mathrm{AMB})+\left(2,46^{*} 1-\mathrm{RM}\right)$ & & & \\
$\mathrm{y} 4=4,40+(14,27 * \mathrm{AMB})+(2,51 * 1-\mathrm{RM})$ & 0,68 & 0,65 & 2,2 \\
\hline
\end{tabular}

$\mathrm{PT}_{\text {ISo, }}$ pico de torque isométrico; $\mathrm{PT}_{\mathrm{CON}}$, pico de torque concêntrico; 1-RM, 1-repetição máxima; $\mathrm{AMB}$, arremesso de medicine ball de $2 \mathrm{~kg}$; IFUNC, índice funcional.

Fonte: Própria autora. 
Tabela 3 - Coeficientes e colinearidade dos modelos propostos para o pico de torque isométrico (PTiso, modelo 1 e 2) e pico de torque extensor concêntrico a $60 \%$ s (PT $\mathrm{CON}$, modelo 3 e 4$)$

\begin{tabular}{|c|c|c|c|c|c|c|c|c|}
\hline \multirow[t]{2}{*}{ Modelo } & \multicolumn{2}{|c|}{$\begin{array}{l}\text { Coeficientes não } \\
\text { padronizados }\end{array}$} & \multirow{2}{*}{$\begin{array}{c}\text { Coeficientes } \\
\text { padronizados } \\
\text { Beta }\end{array}$} & \multicolumn{3}{|c|}{ Intervalo de confiança $95,0 \%$ para B } & \multicolumn{2}{|c|}{ Colinearidade } \\
\hline & $\mathrm{B}$ & Modelo padrão & & $\begin{array}{l}\text { Limite } \\
\text { inferior }\end{array}$ & Limite superior & Tolerância & FIV & $\mathrm{p}$ \\
\hline \multicolumn{9}{|l|}{ Modelo 1} \\
\hline (Constante) & 13,93 & 31,51 & --- & $-49,53$ & 77,38 & & & \\
\hline IFUNC & $-2,23$ & 2,63 & $-0,09$ & $-7,52$ & 3,06 & 0,88 & 1,14 & 0,40 \\
\hline 1-RM & 3,26 & 0,74 & 0,53 & 1,77 & 4,75 & 0,62 & 1,63 & $<0,01$ \\
\hline AMB & 21,85 & 8,44 & 0,30 & 4,85 & 38,84 & 0,67 & 1,49 & 0,013 \\
\hline \multicolumn{9}{|l|}{ Modelo 2} \\
\hline (Constante) & $-6,94$ & 19,66 & --- & $-46,51$ & 32,64 & & & \\
\hline AMB & 21,83 & 8,41 & 0,30 & 4,91 & 38,76 & 0,67 & 1,49 & 0,013 \\
\hline $1-\mathrm{RM}$ & 3,44 & 0,70 & 0,56 & 2,02 & 4,86 & 0,67 & 1,49 & $<0,01$ \\
\hline \multicolumn{9}{|l|}{ Modelo 3} \\
\hline (Constante) & 12,26 & 19,322 & --- & $-26,656$ & 51,176 & & & \\
\hline $1-\mathrm{RM}$ & 2,461 & 0,413 & 0,619 & 1,63 & 3,293 & 0,671 & 1,49 & $<0,01$ \\
\hline AMB & 13,98 & 5,079 & 0,279 & 3,755 & 24,216 & 0,705 & 1,41 & $<0,01$ \\
\hline IFUNC & $-0,781$ & 1,544 & $-0,046$ & $-3,891$ & 2,329 & 0,868 & 1,15 & 0,61 \\
\hline
\end{tabular}

Modelo 4

(Constante)

4,398

11,386

$---$

$-18,52$

27,316 
Silva, KG, Orssatto, LBR, Barros, INM, Nascimento, KS, Streit, IA, Bezerra, ES. Torque muscular de idosos baseado no desempenho físico funcional / Muscle torque of elderly based on physical functional performance

$\begin{array}{lcccccccc}1-\mathrm{RM} & 2,513 & 0,397 & 0,632 & 1,714 & 3,312 & 0,715 & 1,4 & <0,01 \\ \text { AMB } & 14,279 & 5,005 & 0,285 & 4,205 & 24,354 & 0,715 & 1,4 & <0,01\end{array}$

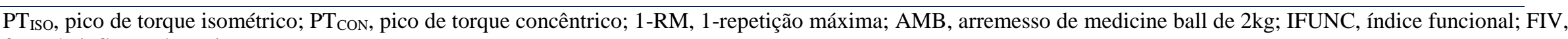
fator de inflação da variação

Fonte: Própria autora. 
O $\mathrm{R}^{2}$ para os modelos no $\mathrm{PT}_{\mathrm{ISO}}$ explicam em $60 \%$ as variações do pico de torque isométrico, assim como, os modelos do $\mathrm{PT}_{\mathrm{CON}}$ podem ser explicados em $68 \%$ das variações. Tais explicações foram confirmadas pelo teste de Durbin-Watson, que apresenta uma satisfação para a hipótese da independência dos erros, com valores muito próximos a 2 (Tabela 2).A análise de variância demonstra que os modelos de regressão são significativamente melhores para prever o PT ISO (modelo 1, F=22,51, $\mathrm{p}<0,001$; modelo 2, F=33,61, $\mathrm{p}<0,001$ ) e PT $\mathrm{CON}$ (modelo 3, F=47,24, p<0,001; modelo 4, F=31,07, $\mathrm{p}<0,001$ ), do que utilizar a média das variáveis previsora.

Os coeficientes padronizados e não padronizados, apresentados na tabela 3 , fornecem a importância de cada preditor. Sendo assim, em todos os modelos que contêm o preditor IFUNC o nível de significância, permite-se desconsiderar este preditor ( $p>0,05)$ e considerar apenas 1 RM e arremesso da bola de medicine ball $(\mathrm{p}<0,01)$. Os valores de VIF indicam a ausência de multicolinearidade (próximos a 1), assim como os valores de tolerância são aceitáveis (maiores que 0,20$)$.

A relação entre o valor previsto padronizado, com o valor do resíduo padronizado, demonstra que todos os modelos satisfazem a hipótese de linearidade (Figura 1). Tal confirmação é amparada pela distribuição normal apresentada em todos os modelos (Figura 2). 
Figura 1 - Relação de linearidade do valor previsto padronizado com o valor do resíduo padronizado para a regressão para PTISO no modelo 1 (painel a) e modelo 2 (painel b); e PTCON no modelo 3 (painel c) e modelo 4 (painel d)

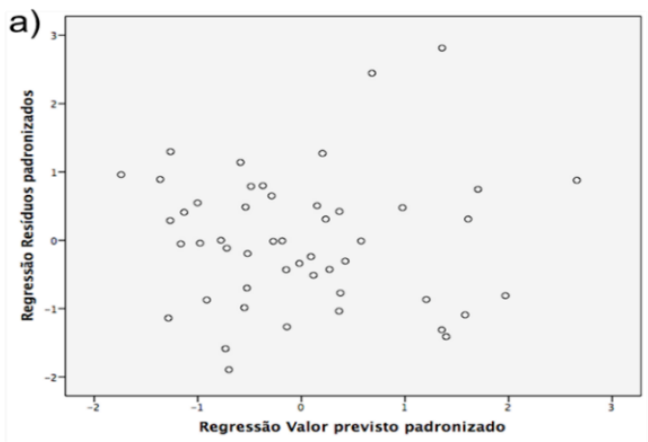

c)

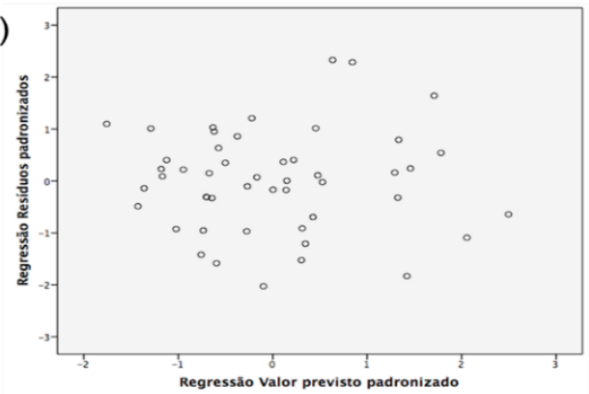

Fonte: Própria autora. b)

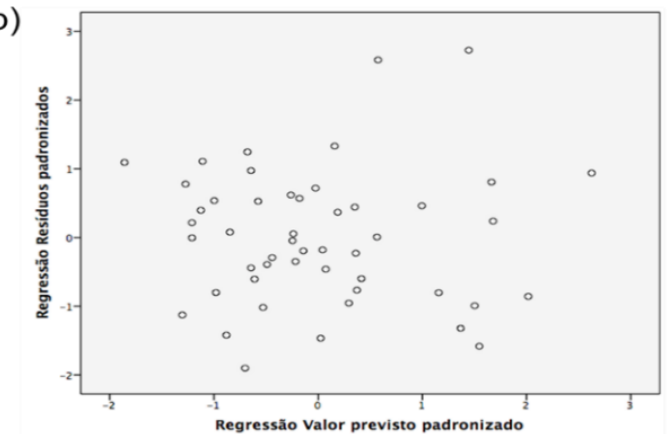

d)

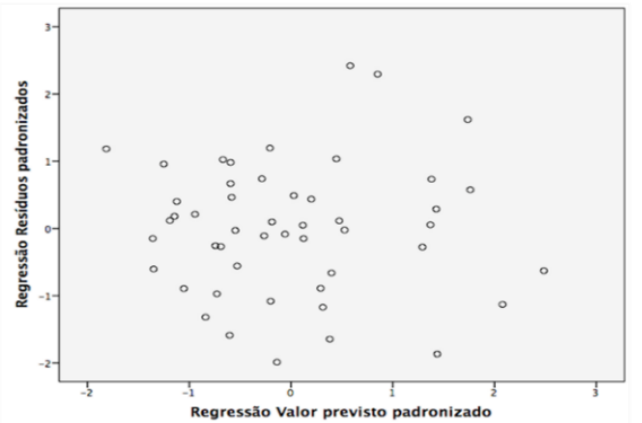

Figura 2 - Histograma da regressão dos resíduos padronizados para PT ISo no modelo 1 (painel a) e modelo 2 (painel b); e PT CON no modelo 3 (painel c) e modelo 4 (painel d)

a)

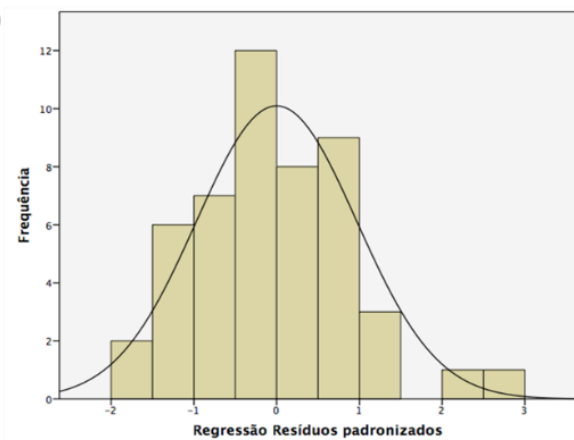

c)

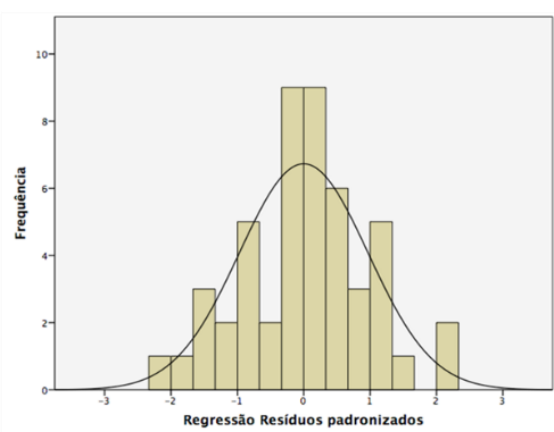

b)

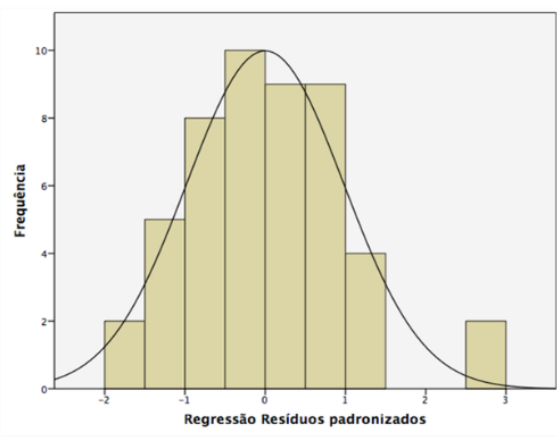

d)

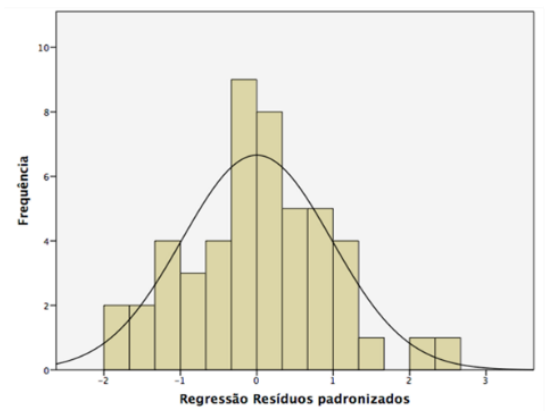

Fonte: Própria autora. 


\section{Discussão}

Os principais resultados deste estudo mostraram que o modelo y2 e y4, os quais continham as variáveis 1-RM e arremesso da bola de medicine ball, explicam respectivamente até $60 \%$ e $68 \%$ a variação do $\mathrm{PT}_{\text {ISO }}$ e $\mathrm{PT}_{\mathrm{CON}}$, de idosos de ambos os sexos. Tais achados demonstram que a variável proveniente dos testes de desempenho físicos funcionais, que simulam atividade da vida diária (subir e descer escadas; e timed up and go), não são capazes de predizer isoladamente o desempenho do $\mathrm{PT}_{\text {ISO }}$ e $\mathrm{PT}_{\mathrm{CON}}$. Contrapondo a hipótese inicial que o desempenho do pico de torque, tanto isométrico, como dinâmico, teria uma relação isolada com o desempenho nos testes físicos funcionais.

A forte correlação entre o PTISO e PTCON com o 1-RM, pode ser explicada pela semelhança cinesiológica entre ambos os testes, realizados para extensores de joelho no dinamômetro isocinético, ou no equipamento tradicional cadeira extensora. $\mathrm{O}$ movimento da cadeira extensora envolve músculos extensores do joelho (20). Esse movimento é uma cadeia cinética aberta, caracterizada pela alta demanda do quadríceps (21) Tal demanda se dá em virtude da carga ser toda movimentada apenas no movimento de extensão do joelho, deixando músculos que usualmente colaborariam em um exercício de cadeia fechada (ex. leg press, e agachamento), como glúteo máximo (22) e isquiotibiais (23) ausente nesta condição estudada. Esse padrão de movimento e recrutamento muscular são similares ao observado durante o teste de extensores de joelho no dinamômetro isocinético, com exceção apenas do tipo de contração adotado (i.e., isocinética ou isotônica).

Testes de desempenho físico funcionais têm sido avaliados amplamente para o acompanhamento da melhora do desempenho de idosos após períodos de intervenção com programas de treinamento $(12,24,25)$. Apesar de diversos estudos terem reportado melhoras significativas do desempenho físico funcional de idosos, após períodos de treinamento, ainda não está claro na literatura a influência dos ganhos de força muscular, sobre os ganhos de desempenho físico funcional. Todavia, tem sido verificado relação entre testes desempenho físico funcional e medidas de força muscular (26). O teste de equilíbrio dinâmico e mobilidade (ex. teste timed up and go) pode ser um indicador de disfunção neuromuscular. Com o avançar da idade mostram-se respostas negativas para receptores sensoriais e nos nervos periféricos, associadas à diminuição da acuidade visual e da função vestibular. Isso afeta o controle postural e também a produção de força muscular nos membros inferiores, levando à redução do desempenho da marcha, da mobilidade e, consequentemente, do equilíbrio postural (27). Os resultados do presente estudo indicam que esse tipo de teste pouco influenciou em medidas de 
força muscular do membro inferior, o que corrobora com achados de que testes de desempenho físico funcionais não apresentam marcante relação para medidas de força muscular dos membros inferiores (28).

Nossos modelos apresentaram forte associação entre o teste de arremesso da bola de medicine ball e o teste isométrico e dinâmico de extensão do joelho no isocinético. Similarmente, o teste do membro superior (ex. preensão manual) tem sido associado à melhora do desempenho em testes realizados no isocinético em idosos (29) e esses estão positivamente correlacionados com o estado de saúde do indivíduo, principalmente quando são apresentados de forma combinada (30).

Embora não se tenha usado a força de preensão manual, a medida utilizada no presente estudo, arremesso da medicine ball apresenta alta reprodutibilidade. Ela é uma medida barata e segura para avaliar a potência do membro superior em idosos(31). Apesar dessas características, a inclusão deste teste específico foi de cunho exploratório, partindo do princípio que a medida de preensão manual é um bom preditor para desempenho de força muscular do membro inferior, como confirmado anteriormente (29).

Nessa perspectiva, os modelos preditivos 2 e 4 propostos evidenciam que testes clínicos, de fácil aplicação, e com boa reprodutibilidade (1-RM e arremesso da bola de medicine ball) podem ser usados como indicadores de mudanças no pico de torque isométrico e dinâmico de idosos. Hoje, a literatura evidencia que adaptações neuromusculares com a idade resultam em uma velocidade reduzida de contração muscular, levando a uma perda acelerada de potência muscular e força rápida em comparação com a força isométrica; tal condição coloca os idosos em maior risco de quedas(2). De maneira simplificada, essa redução da capacidade de produzir força máxima e rápida é influenciada, principalmente, pela redução da velocidade e intensidade de condução do sinal elétrico pelo sistema nervoso (unidade motora de rápida fadiga, FF), pela exacerbada atrofia e apoptose das fibras do tipo IIX. Portanto, o monitoramento da capacidade de produção de força muscular em idosos é fundamental para um envelhecimento saudável, proporcionando o acompanhamento dos resultados de intervenções. Dessa maneira, o presente estudo proporcionou uma estratégia de baixo custo para estimar o $\mathrm{PT}_{\text {ISO }}$ e $\mathrm{PT}_{\mathrm{CON}}$ de idosos, o que tem alta validade para profissionais da área (e.g., personal trainers, fisioterapeutas, nutricionistas e reumatologistas).

Os pontos fortes e as limitações do presente estudo devem ser relatados. Usar o mesmo avaliador experiente para realizar todos os testes deu ao nosso estudo, alta validade interna. Outra ponto positivo do estudo é que usamos um número de sujeitos adequado e uma população homogênea de idosos desacostumados ao treinamento resistido. Além do que, os resultados 
fornecem informações valiosas sobre os tipos de testes de força muscular, que mostraram maior correlação com medidas isométricas e dinâmicas no isocinético (avaliação padrão ouro para medida de capacidade muscular). Por outro lado, sugere-se cautela com a aplicação das equações preditivas no diagnóstico dos fatores dependentes ( $\mathrm{PT}_{\text {ISO }}$ e $\mathrm{PT}_{\mathrm{CON}}$ ), pois ela deve ser testada com uma amostra independente com mesma idade. Isso nos leva a recomendar que os resultados não devam ser extrapolados para indivíduos previamente treinados, idosos longevos (e.g., >80 anos) ou frágeis. Algumas fraquezas devem ser consideradas, como: reduzido número de homens (apenas 10), o que sugerimos ampliação em futuras intervenções de mesma similaridade; ausência de teste para captação do pico de torque de músculo dos segmentos superiores e embora nossa amostra tenha apresentado um poder adequado, um aumento desta em futuros trabalhos pode potencializar o poder preditor, não só dos testes de desempenho funcional, mas da equação de forma generalizada.

Em suma, a força dinâmica máxima avaliada por meio do teste de 1-RM na cadeira extensora e a potência muscular de membro superior quando associadas, explicam em até $60 \%$ da variação entre sujeitos para o PTISO e PTCON de extensores de joelho em idosos não treinados. Dessa maneira, a associação de testes de baixo custo, como o 1-RM e o arremesso da bola de medicine ball, podem ser usados para monitoramento do $\mathrm{PT}_{\text {ISO }}$ e $\mathrm{PT}_{\mathrm{CON}}$ quando não houver disponibilidade de avaliação por meio do padrão ouro (i.e., dinamômetro isocinético).

\section{Agradecimentos}

A Fundação de Amparo à Pesquisa do Estado do Amazonas por conceder bolsa de iniciação científica a Keuly Garcia da Silva (RESOLUÇÃO N. 012/2018 - PAIC-AM 2018 UFAM). Ao Conselho Nacional de Desenvolvimento Científico e Tecnológico (CNPQ) e ao Conselho de Aperfeiçoamento de Pessoal de Nível Superior (CAPES) por darem suporte ao Programa de Pós Graduação Stricto Sensu em Ciências do Movimento Humano, Faculdade de Educação Física e Fisioterapia-Universidade Federal do Amazonas. 


\section{Referências}

1. Enoka RM. Morphological features and activation patterns of motor units. J Clin Neurophysiol. 1995;12(6):538-59.

2. Orssatto LB da R, Wiest MJ, Diefenthaeler F. Neural and musculotendinous mechanisms underpinning age-related force reductions. Mech Ageing Dev . 2018;175:17-23.

3. Raj IS, Bird SR, Shield AJ. Aging and the force-velocity relationship of muscles. Exp Gerontol. 2010 Feb;45(2):81-90.

4. Lindle RS, Metter EJ, Lynch NA, Fleg JL, Fozard JL, Tobin J, et al. Age and gender comparisons of muscle strength in 654 women and men aged 20-93 yr. J Appl Physiol . 1997;83(5):1581-7.

5. Reid KF, Fielding RA. Skeletal muscle power: a critical determinant of physical functioning in older adults. Exerc Sport Sci Rev . 2012 ;40(1):4-12.

6. Hunter SK, Pereira HM, Keenan KG. The aging neuromuscular system and motor performance. J Appl Physiol (Bethesda, Md 1985) . 2016 ;121(4):982-95.

7. Pion CH, Barbat-Artigas S, St-Jean-Pelletier F, Chevalier S, Gaudreau P, Gouspillou G, et al. Muscle strength and force development in high- and low-functioning elderly men: Influence of muscular and neural factors. Exp Gerontol . 2017;96:19-28. Available from: https://pubmed.ncbi.nlm.nih.gov/28579089/

8. Miles J, Shevlin M. Applying regression and correlation : a guide for students and researchers. Sage Publications; 2001. 253 p.

9. Elias LJ, Bryden MP, Bulman-Fleming MB. Footedness is a better predictor than is handedness of emotional lateralization. Neuropsychologia. 1998;36(1):37-43.

10. Orssatto LB da R, Bezerra E de S, Moura BM de, de Castro JAC, Silva DAS, Moro ARP, et al. Estimating bone mineral content based on different types of muscle strength tests. $\mathbf{J}$ Bodyw Mov Ther. 2018;22(3):586-91.

11. Orssatto LB da R, Moura BM De, Sakugawa RL, Radaelli R, Diefenthaeler F. Leg press exercise can reduce functional hamstring:quadriceps ratio in the elderly. J Bodyw Mov Ther . 2018;22(3):592-7.

12. Lima AB, de Souza Bezerra E, da Rosa Orssatto LB, de Paiva Vieira E, Picanço LAA, Dos Santos JOL. Functional resistance training can increase strength, knee torque ratio, and functional performance in elderly women. J Exerc Rehabil . 2018 Aug;14(4):654-9.

13. Brown LE, Weir JP. ASEP procedures recommendation I: Accurate assessment of muscular strength and power. J Exerc Physiol Online. 2001;4(3):1-21.

14. Pereira A, Izquierdo M, Silva AJ, Costa AM, González-Badillo JJ, Marques MC. Muscle performance and functional capacity retention in older women after high-speed power training cessation. Exp Gerontol. 2012;47(8):620-4. 
15. Butler AA, Menant JC, Tiedemann AC, Lord SR. Age and gender differences in seven tests of functional mobility. J Neuroeng Rehabil . 2009 Jul 30;6(6):31.

16. Rikli RE, Jones CJ. Development and validation of criterion-referenced clinically relevant fitness standards for maintaining physical independence in later years. Gerontologist.

2013;53(2):255-67.

17. Bennell K, Dobson F, Hinman R. Measures of physical performance assessments: SelfPaced Walk Test (SPWT), Stair Climb Test (SCT), Six-Minute Walk Test (6MWT), Chair Stand Test (CST), Timed Up \& Go (TUG), Sock Test, Lift and Carry Test (LCT), and Car Task. Arthritis Care Res. 2011;63(SUPPL. 11):350-70.

18. Silva ME da, Orssatto LB da R, Bezerra E de S, Silva DAS, Moura BM de, Diefenthaeler $\mathrm{F}$, et al. Reducing measurement errors during functional capacity tests in elders. Aging Clin Exp Res. 2017;In Press(0):0.

19. Cohen J. Statistical power analysis for the behavioral sciences. L. Erlbaum Associates; 1988. 567 p.

20. Escamilla, RF.; Fleisig, GS.; Zheng, N; Barrentine, SW.; Wilk, KE.; Andrews JR. Biomechanics of the Knee during Closed Kinetic Chain and Open Kinetic Chain Exercises. Med Sci Sport Exerc. 1998;30(4):556-69.

21. Akima H, Saito A. Activation of quadriceps femoris including vastus intermedius during fatiguing dynamic knee extensions. Eur J Appl Physiol. 2013;113(11):2829-40.

22. Da Silva, EM, Brentano, MA, Cadore, EL, De Almeida, APV, and Kruel L. Analysis of muscle activation during different leg press exercises at submaximum effort levels. Jounal Strength Cond Res. 2008;22(4):1059-65.

23. Wilk KE, Escamilla RF, Fleisig GS, Barrentine SW, Andrews JR, Boyd ML. A comparison of tibiofemoral joint forces and electromyographic activity during open and closed kinetic chain exercises. Am J Sports Med. 1996;24(4):518-27.

24. Bezerra E de S, Orssatto LB da R, de Moura BM, Willardson JM, Simão R, Moro ARP. Mixed Session Periodization as a New Approach for Strength, Power, Functional Performance, and Body Composition Enhancement in Aging Adults. J strength Cond Res . 2018;32(10):2795-806.

25. da Rosa Orssatto LB, de Moura BM, de Souza Bezerra E, Andersen LL, de Oliveira SN, Diefenthaeler F. Influence of strength training intensity on subsequent recovery in elderly. Exp Gerontol . 2018;106:232-9.

26. Moura BM, Sakugawa RL, Orssatto LBR, de Lima LAP, Pinto RS, Walker S, et al. Functional capacity improves in-line with neuromuscular performance after 12 weeks of nonlinear periodization strength training in the elderly. Aging Clin Exp Res . 2018;30(8):959-68.

27. Serra MM, Alonso AC, Peterson M, Mochizuki L, Greve JMD, Garcez-Leme LE. Balance and Muscle Strength in Elderly Women Who Dance Samba. PLoS One .

2016;11(12):e0166105. 
28. Chiles Shaffer N, Fabbri E, Ferrucci L, Shardell M, Simonsick EM, Studenski S. Muscle Quality, Strength, and Lower Extremity Physical Performance in the Baltimore Longitudinal Study of Aging. J frailty Aging . 2017;6(4):183-7.

29. Alonso AC, Ribeiro SM, Luna NMS, Peterson MD, Bocalini DS, Serra MM, et al. Association between handgrip strength, balance, and knee flexion/extension strength in older adults. PLoS One . 2018;13(6):e0198185.

30. Chan OYA, van Houwelingen AH, Gussekloo J, Blom JW, den Elzen WPJ. Comparison of quadriceps strength and handgrip strength in their association with health outcomes in older adults in primary care. Age (Omaha) . 2014; 36(5):9714.

31. Harris C, Wattles AP, Debeliso M, Sevene-Adams PG, Berning JM, Adams KJ. The seated medicine ball throw as a test of upper body power in older adults. J Strength Cond Res . 2011; 25(8):2344-8. 